\title{
Bone mineral density in patients with juvenile systemic lupus erythematosus
}

\author{
Ana Paula Roenick Guenka*, Blanca Bica, Laura Mendonça, Lucas Velloso, Mario Leitão \\ From 18th Pediatric Rheumatology European Society (PReS) Congress \\ Bruges, Belgium. 14-18 September 2011
}

\section{Background}

Cross-sectional study, in which were evaluated the bone mineral density of 53 patients from the Rheumatology ambulatory, diagnosed with JSLE in their childhood or teenage years, according to American College of Rheumatology criteria.

\section{Aim}

Evaluate the bone mass in a group of patients diagnosed with juvenile systemic lupus erythematosus (JSLE), which carry high risks of developing low bone mass, as well as verifying alterations on calcium metabolism.

\section{Method}

Cross-sectional study, in which were evaluated 53 patients from the Rheumatology ambulatory in HUCFF/ UFRJ, diagnosed with JSLE in their childhood or teenage years, according to ACR (American College of Rheumatology) criteria. Subjects were submitted to a single blood and urine collection for dosing of the following variables: alkaline phosphatase, serum phosphorus and calcium, urea, creatinine, erythrocyte sedimentation rate (ESR), intact PTH, free T4, TSH, 24-hour calciuria. Bone density was measured from lumbar spine, and full body and/or total femur, according to age range, using DEXA methods and Prodigy Advance equipment. Also, medical records were analyzed collecting the following data: age, sex, time coping with the disease, body mass index (BMI), disease progression with SLEDAI average for the last 6 months, number of diagnostic criteria, cumulative dose of 3 drug-induced osteoporosis classes (glucocorticoid, methotrexate and anticoagulant). Finally, the collected data was submitted to statistic analysis. Patients suffering from renal insufficiency or currently on bone antiresorptive or bone formation stimulators drugs were excluded.

\section{Results}

$92 \%$ of patients were females, being only 4 male subjects. Analyzed parameters averages, and their corresponding standard deviation (SD): Age -21 years old $(4,0)$; time coping with the disease $-7,9$ years $(5,7)$; number of ACR criteria at diagnosing moment $-5,6$ $(1,4)$; BMI $-23,2 \mathrm{~kg} / \mathrm{m}^{2}(4,7)$; SLEDAI - 3,7 $(4,6)$ $\mathrm{R}=1,23$. Not only was the accumulative glucocorticoid dosage calculated, but also the prednisone equivalency. The maximum used dosage was $133,88 \mathrm{~g}$, with an average of $29,1 \mathrm{~g}$ and standard deviation of $27,8(\mathrm{R}=1,0)$. The only anticoagulant used was warfarin, with maximum dosage of $39,76 \mathrm{~g}$, average of $1,3 \mathrm{~g}$ and standard deviation of 5,9 $(R=4,5)$. Maximum dosage for methotrexate was $2,49 \mathrm{~g}$, average of $0,2 \mathrm{~g}$ and standard deviation of 0,6 $(\mathrm{R}=2,6) .15$ patients presented densitometry alterations, characterized by Z-score lower or equal to 2 standard deviation. No clinically significant laboratorial alterations on calcium metabolism were found.

\section{Conclusion}

No direct or inverse linear relation (correlation) was observed between the analyzed parameters and densitometry findings according to the Z-score.

Published: 14 September 2011

\section{doi:10.1186/1546-0096-9-S1-P253}

Cite this article as: Guenka et al:: Bone mineral density in patients with juvenile systemic lupus erythematosus. Pediatric Rheumatology 20119 (Suppl 1):P253. 